\title{
LHCb Detector and Performance
}

\section{Neville Harnew* ${ }^{* \dagger}$}

The University of Oxford

E-mail: n.harnew1@physics.ox.ac.uk

The LHCb experiment at the CERN Large Hadron Collider (LHC) will measure the CKM parameters of the Unitarity Triangle with high precision, and is designed to discover physics beyond the Standard Model in rare heavy-flavour decays. The experiment is configured in the forward direction and covers a unique rapidity range at the LHC, $1.9<\eta<4$.9. This paper describes the detector components of $\mathrm{LHCb}$ and the operating performances, focusing on the vertex locator, particle identification, calorimetry and muon system. In 2010, LHCb recorded a total of 37.7 $\mathrm{pb}^{-1}$ of data at $\sqrt{s}=7 \mathrm{TeV}$ with a data-taking efficiency of better than $90 \%$. A selection of LHCb physics results will be shown, and the prospects for data taking in 2011 will be discussed.

The 13th International Conference on B-Physics at Hadron Machines - Beauty2011, April 04-08, 2011

Amsterdam, The Netherlands

\footnotetext{
* Speaker.

On behalf of the LHCb Collaboration.
} 


\section{Introduction}

The exploitation of $B$ physics in the high rapidity (forward) regions at hadron colliders has historically been challenging. Whilst experiments at the CERN ISR paved the way for forward physics measurements, the SPS and Tevatron focussed on the central, high $p_{T}$, region. The $\mathrm{H} 1$ and ZEUS experiments at HERA [1] demonstrated that high track multiplicities and secondary interactions in the forward region could make full event reconstruction difficult. A dedicated forward $B$-physics experiment, HERA-B, suffered numerous problems [2].

The LHCb experiment [3], shown in Fig. 1, is a forward spectrometer, operating at the CERN $p p$ Large Hadron Collider (LHC). LHCb is optimized for the study of heavy-flavour hadron production at the LHC, for which the angular distribution is predominantly forward-backward peaked. The experiment will measure the CKM parameters of the Unitarity Triangle with high precision, and is designed to discover physics beyond the Standard Model in rare heavy-flavour decays. The spectrometer covers the rapidity range $1.9<\eta<4.9$, which is unique amongst the LHC experiments. Despite LHCb covering only $\sim 4 \%$ of the solid angle, the experiment captures approximately $40 \%$ of the heavy-quark production cross section.

The major detector elements of LHCb comprise a silicon VErtex LOcator (VELO), a dipole magnet providing an integrated field of $\sim 4 \mathrm{Tm}$, two RICH detectors, a system of tracking chambers, electromagnetic (ECAL) and hadronic (HCAL) calorimeters and a muon system. In the forward region covered by $\mathrm{LHCb}$, the track occupancies are dense and secondary interactions must be minimized. Triggering the detector in this environment is a significant challenge.

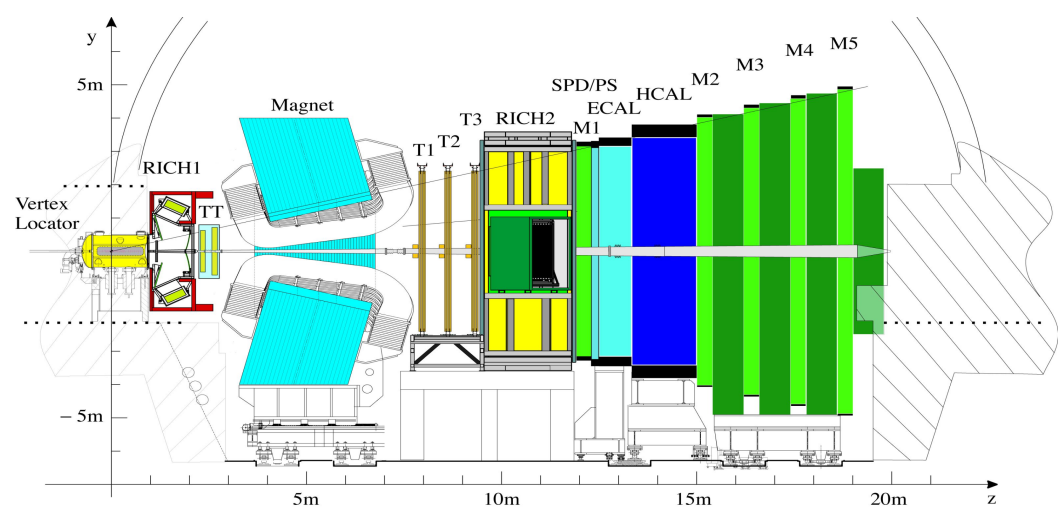

Figure 1: A schematic of the LHCb experiment.

\section{The LHCb 2010 running conditions}

Figure 2 (left) shows the time evolution during 2010 of the integrated luminosity recorded by $\mathrm{LHCb}$ at a centre of mass energy of $\sqrt{s}=7 \mathrm{TeV}$. A total of $37.7 \mathrm{pb}^{-1}$ was recorded. However operation of the detector was not always straightforward. LHCb was designed to run at a luminosity of $2 \times 10^{32} \mathrm{~cm}^{-2} \mathrm{~s}^{-1}$ with no more than a single $p p$ interaction per bunch crossing. To maximize the number of single interactions, a mean number of interactions per bunch, $\langle\mu\rangle \sim 0.4$, is optimal. 
At end of the 2010 LHC running period, the peak instantaneous luminosity was almost at the design value of $2 \times 10^{32} \mathrm{~cm}^{-2} \mathrm{~s}^{-1}$. However this luminosity was delivered in only 344 colliding bunches (i.e. only $\sim 1 / 8^{\text {th }}$ of the bunches were filled). Hence the experiment had to cope with $\mathrm{a}\langle\mu\rangle$ value which was significantly greater than design. This is demonstrated in Fig. 2(right) which shows $\langle\mu>$ rising as high as 2.5 . Nevertheless, even in these difficult conditions, the data-taking efficiency was better than $90 \%$ and all sub-detectors operated at typically $>99 \%$ efficiency.
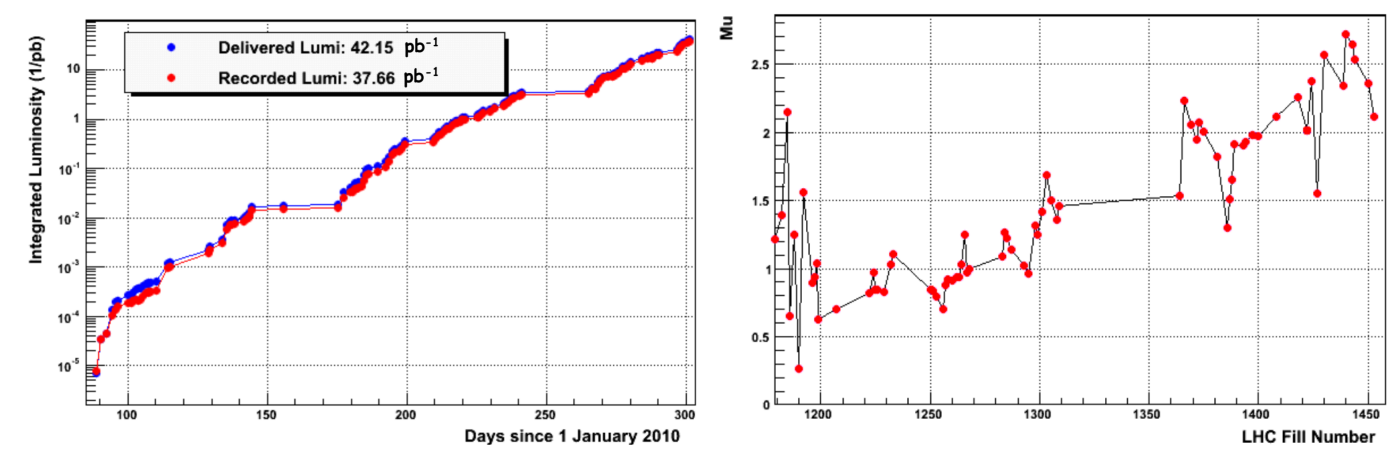

Figure 2: (Left) The time evolution of the integrated luminosity recorded by LHCb in 2010; (Right) the mean number of interactions, $\langle\mu\rangle$, as a function of LHC fill number during this period.

\section{The $\mathrm{LHCb}$ detector performance}

The LHCb detector has several key components. The performance of the trigger, the tracking components, the RICH particle identification system, the calorimeters and the muon chambers are discussed below.

The first stage of the LHCb trigger is the Level-0 (L0) which is made from a high- $p_{T}$ ECAL or HCAL cluster(s), or muon candidate(s). The maximum input rate into the L0 is at the LHC bunchcrossing frequency, $40 \mathrm{MHz}$. The second stage is the High-Level Trigger (HLT), implemented in a processor farm, and separated into HLT 1 and HLT 2. The HLT 1 associates L0 signals with tracks, in particular those VELO tracks which are displaced from the primary vertex. The maximum input rate into HLT 1 is $1 \mathrm{MHz}$. At the HLT 2 stage the full detector information is available. Inclusive and exclusive signatures can be selected in many key channels. There is a maximum output rate of $2 \mathrm{kHz}$ from HLT 2 onto disk.

To cope with the increased number of primary interactions, the trigger strategy had to evolve over the course of the run, whilst always retaining good efficiency. In the 2010 data, L0 $\times$ HLT efficiencies were determined from the data themselves using so-called "tag and probe" methods. The single muon trigger efficiency, determined from $J / \psi$ decays, was found to be $94.9 \pm 0.2 \%$ (compared to the efficiency measured in Monte Carlo of $93.3 \pm 0.2 \%)$. The hadron trigger efficiency, determined from $D^{0}$ decays, was found to be $60 \pm 4 \%$ (compared to Monte Carlo of $\sim 66 \%$ ).

The LHCb tracking system comprises the VELO for determining primary and secondary vertices, the dipole magnet, and four tracking stations. The stations employ silicon detectors and straw tubes in the outer regions. The performance of the tracking system and its alignment is 
demonstrated in Fig. 3. This shows the evolution of the $J / \psi \rightarrow \mu^{+} \mu^{-}$mass measurement as a function of time. From May 2010 to December 2010 the $J / \psi$ mass resolution steadily improved from 17.1 MeV to $13.3 \mathrm{MeV}$, compared to the Monte-Carlo expectation of $12.1 \mathrm{MeV}$. This demonstrates that the detector alignment is well understood.

The vertex resolution and $B$-hadron lifetime performance of the VELO are close to expectations. The impact parameter resolution is $\sim 15 \mathrm{~mm}$ for the highest $p_{T}$ bins, which is dominated by multiple scattering rather than detector mis-alignment. The proper time resolution for $B$ hadron decays is typically $50 \mathrm{fs}$, which is channel dependent.

Particle identification (PID) is crucial to isolate $B$ decays which have an identical topology, e.g. $B^{0} \rightarrow \pi^{+} \pi^{-}$and $B^{0} \rightarrow K^{+} \pi^{-}$. PID is also vital for flavour tagging. LHCb has two RICH detectors which employ three radiators: aerogel, $\mathrm{C}_{4} \mathrm{~F}_{10}$ and $\mathrm{CF}_{4}$. This combination of radiators provides $\pi / K$ separation between $\sim 2$ to $100 \mathrm{GeV} / \mathrm{c}$. Figure 4 shows the efficiencies and the mis-identification fractions of kaons, determined using data, and compared to Monte Carlo. This performance is established using calibration samples of $K_{s}^{0} \rightarrow \pi^{+} \pi^{-}, \Lambda^{0} \rightarrow p \pi^{-}, D \rightarrow K \pi$ and $\phi \rightarrow K^{+} K^{-}$ decays, where the kaon and pion candidates can be inferred with high purity without recourse to RICH information. It can be seen from Fig. 4 that a kaon is identified as a kaon with a purity of $\sim 95 \%$, averaged over the momentum range, with a corresponding $\sim 7 \%$ fake ID rate. Figure 5 demonstrates the power of the PID system in reducing the background in the $\phi \rightarrow K^{+} K^{-}$channel, measured in an early $\sqrt{s}=900 \mathrm{GeV}$ data sample.

The LHCb calorimeter system employs a Shashlik construction (ECAL) and an iron plus scintillator-tile geometry (HCAL). The ECAL provides photon and electron identification and, together with the HCAL, contributes to the L0 trigger decision. The ECAL also provides the sole reconstruction of $\pi^{0}$ s. The excellent ECAL mass resolution is demonstrated in Fig. 6 for the $\pi^{0} \rightarrow \gamma \gamma$ reconstruction. The measured $\pi^{0}$ mass resolution of $\sigma=7.2 \mathrm{MeV}$ exceeds expectations.

The muon system consists of five stations, M1 to M5. The muon stations employ wire proportional chambers with triple-GEM detectors in the innermost region of M1. The efficiency of single muon identification is determined from data employing a tag and probe method using $J / \psi \rightarrow \mu^{+} \mu^{-}$events. The resulting efficiency is found to be better than $90 \%$ for $p>10 \mathrm{GeV}$. Misidentification probabilities $K \rightarrow \mu, \pi \rightarrow \mu$ and $p \rightarrow \mu$ are also determined from data and are found to be less than $2 \%$ in the same momentum range. Good agreement between data and simulation is observed.
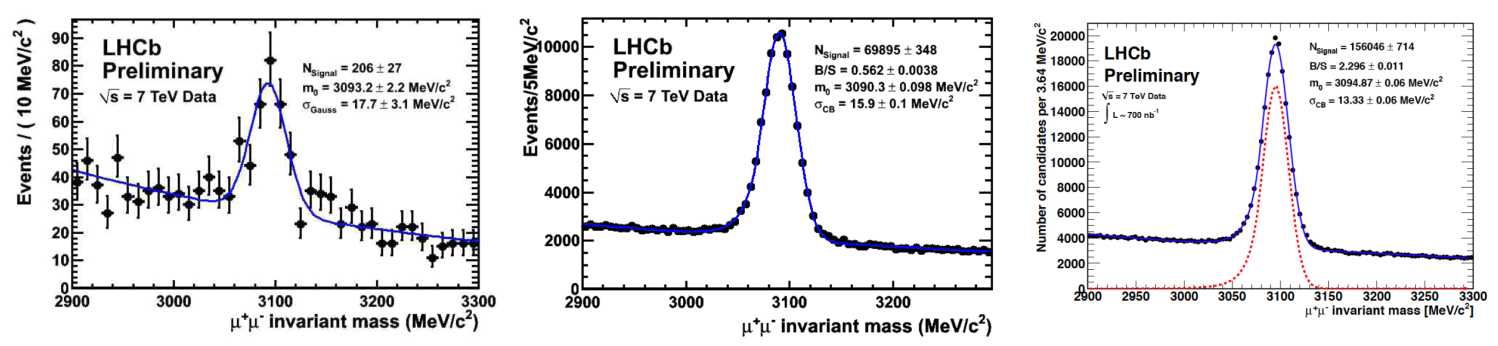

Figure 3: The time evolution of the $J / \psi \rightarrow \mu^{+} \mu^{-}$mass resolution: (Left) the $J / \psi$ mass in May 2010 with a resolution of 17.1 MeV; (Centre) 15.9 MeV in August 2010; (Right) 13.3 MeV in December 2010. The Monte-Carlo expectation is $12.1 \mathrm{MeV}$. 

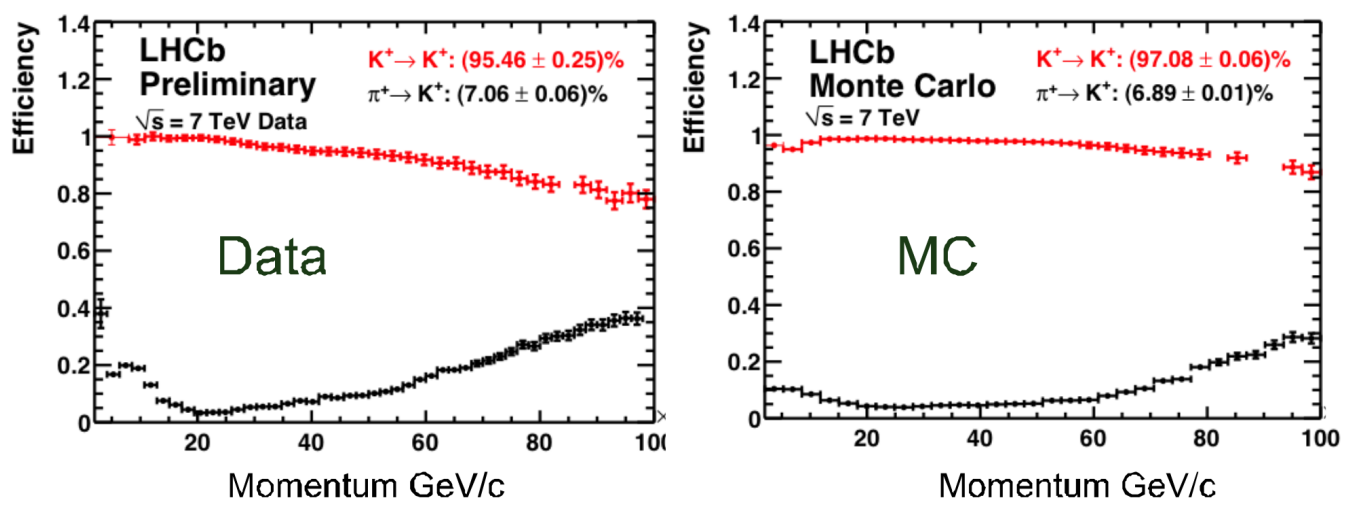

Figure 4: The efficiencies and mis-identification fractions of kaons: (Left) determined from data as a function of momentum using calibration samples described in the text; (Right) Monte Carlo expectation.
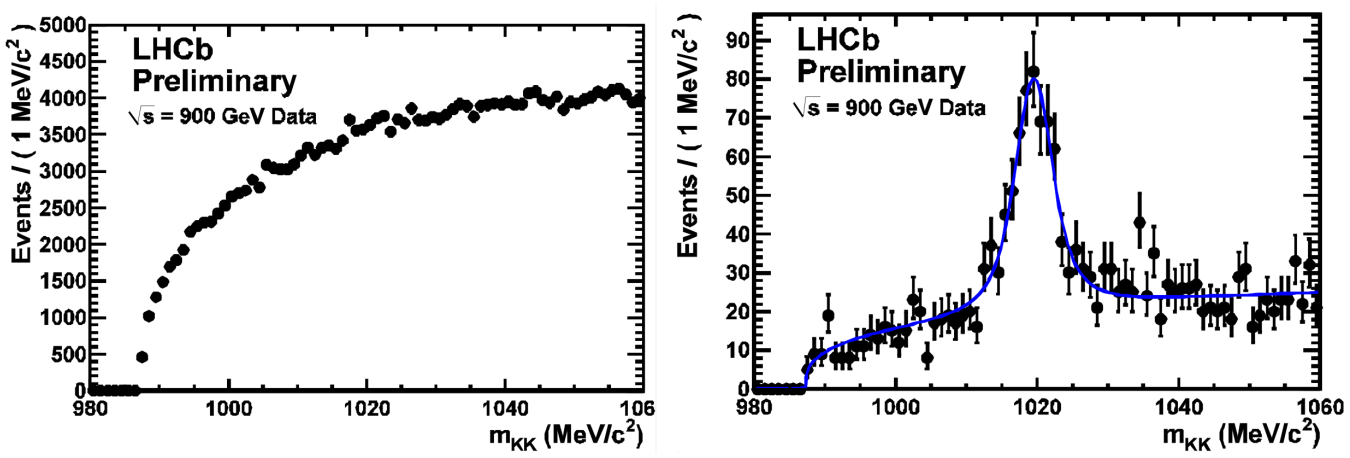

Figure 5: The $K^{+} K^{-}$mass distribution: (Left) without particle identification; (Right) with a RICH selection on each kaon track $(K-\pi \log$-likelihood difference for the $K$ hypothesis $>15)$. The $\phi$ peak is clearly seen.

\section{Selected early physics measurements}

The major physics aims of LHCb are detailed in Ref [4]. Key measurements include:

- Tree and penguin-level determinations of the CKM angle $\gamma$;

- Charmless charged two-body $B$ decays;

- The $B_{s}$ mixing phase $\phi_{s}$ from $B_{s} \rightarrow J / \psi \phi$ decays;

- The branching fractions of $B_{s}$ and $B_{d} \rightarrow \mu^{+} \mu^{-}$;

- The angular analysis of $B^{0} \rightarrow K^{*} \mu^{+} \mu^{-}$decays;

- $B_{s} \rightarrow \phi \gamma$ and similar penguin radiative decays.

All these topics are reported in the proceedings of this conference. Highlighted below is the LHCb tagging performance, the first evidence for direct $\mathrm{CP}$ violation at $\mathrm{LHCb}$, and the $W$ boson production asymmetry. The latter analysis was not envisaged in LHCb's initial physics programme. 


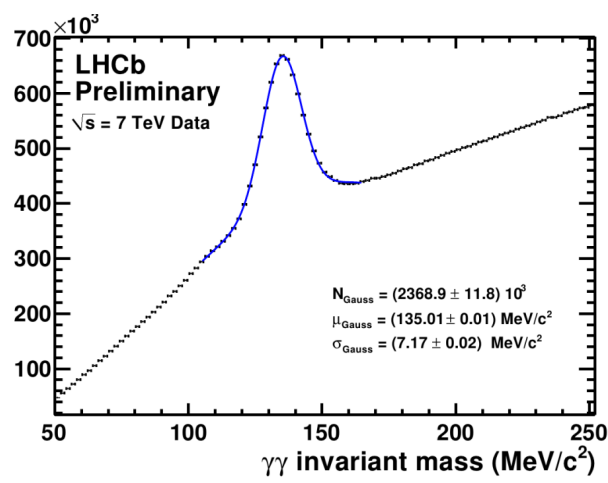

Figure 6: The mass reconstruction of $\pi^{0} \rightarrow \gamma \gamma$ decays measured in the ECAL.

A good tagging performance is vital to any $\mathrm{CP}$-violation measurement where the flavour of the $B$ or $\bar{B}$ hadron being analysed is not uniquely defined by its own decay products. A number of tagging methods have been studied to date: the opposite-side $B$-hadron leptonic charge, the $b \rightarrow c \rightarrow s$ kaon charge and the vertex charge taggers, and the same-side pion charge tagger [5]. The tagging efficiency (or "power") determined via any given method is defined by $\varepsilon_{\text {eff }}=\varepsilon_{\text {tag }}(1-$ $2 \omega)^{2}$, where $\varepsilon_{\text {tag }}$ is the efficiency to tag the $B$ hadron and $\omega$ is the wrong-tag fraction. The "flavour specific" channel $B^{0} \rightarrow D^{*-} \mu^{+} v_{\mu}$ can be used both to measure $B^{0}$ oscillations and also to study the tagging performance (with the aid of charged- $B$ calibration channels). The tagging power measured is $(1.97 \pm 0.18) \%$ using only the opposite-side tagging combinations. An improved performance can be expected following further study.

An example of a direct CP violation measurement is the difference in event rates of $\bar{B}^{0} \rightarrow$ $K^{-} \pi^{+}$and $B^{0} \rightarrow K^{+} \pi^{-}$[6]. This is an analysis in which PID is essential in separating the samples of $\bar{B}^{0}$ and $B^{0}$. A preliminary LHCb measurement gives a $>3 \sigma$ observation of direct CP violation in these channels, although corrections for production and detector asymmetries have yet to be performed. Central $B$ mass values are consistent with expectations. A number of first observations of rare $B_{s}$ decay modes have also been made: $B_{s} \rightarrow D_{s 2}^{+}(2573) \mu^{-} \bar{v}_{\mu}$ [7], $B_{s} \rightarrow J / \psi f_{0}(980)$ [8], $B_{s} \rightarrow K^{* 0} K^{* 0}$ [9] and $\bar{B}_{s} \rightarrow D^{0} K^{* 0}$ [10]. LHCb also has a vibrant programme of charm physics [11].

Finally, there have been several physics topics that were not in our initial planning and, given the unique rapidity range of the $\mathrm{LHCb}$ detector, explorations can be made which are not available to other experiments. An example of such a measurement is the vector boson $W^{+} / W^{-}$production asymmetry. Figure 7 (left) shows the numbers of $W^{-}$and $W^{+}$decaying to $\mu v$ as a function of muon transverse momentum. Here a single isolated $\mu$ is selected with $p_{T}>20 \mathrm{GeV} / \mathrm{c}$, with only small $p_{T}$ in the opposite hemisphere. Figure 7 (right) shows the corresponding charge asymmetry as a function of pseudorapidity, and comparing with next to leading order parton distributions. It can be seen that these measurements are very sensitive to the parton density functions which opens up new regions of $\left(x, Q^{2}\right)$ for proton structure measurements.

\section{The next steps}

At the time of Beauty 2011, the 2011 data-taking run had just started. In a relatively trouble- 

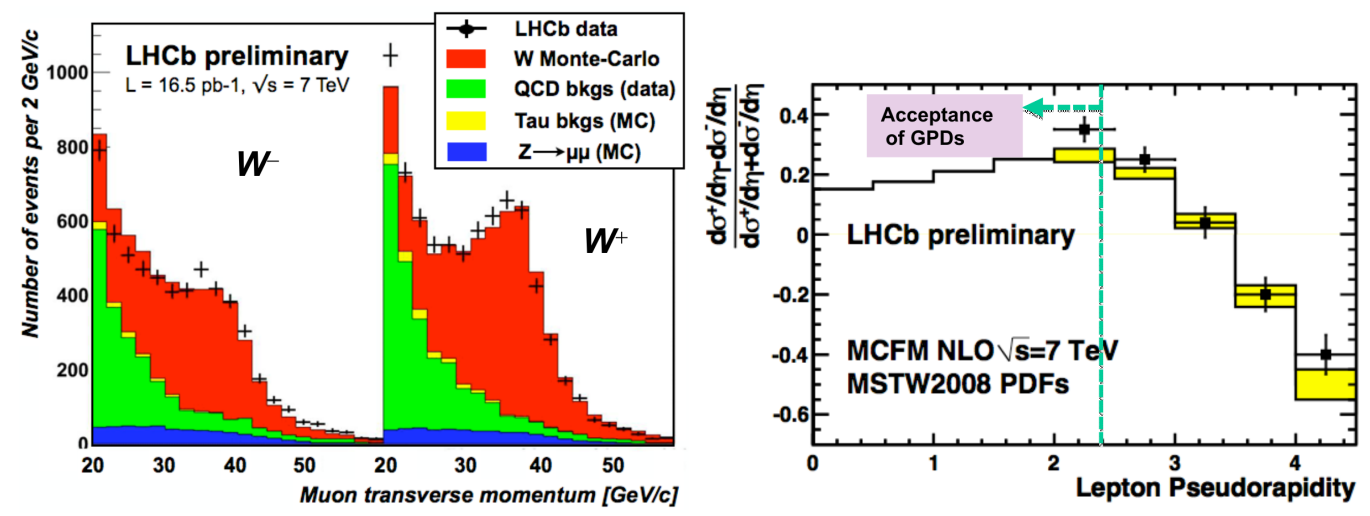

Figure 7: (Left) The numbers of $W^{-}$and $W^{+}$decaying to $\mu v$ as a function of muon transverse momentum; (Right) the $W^{+} / W^{-}$asymmetry as a function of pseudorapidity, showing comparisons with next to leading order parton distributions and also the pseudorapidity regions covered by ATLAS and CMS.

free start-up period, the running conditions experienced by $\mathrm{LHCb}$ at the end of 2010 were on the way to being surpassed. The data-taking efficiency was again better than $90 \%$.

The initial plan in 2011 is to run at a mean number of interactions per bunch-crossing up to $<$ $\mu>=2$. However, it is hoped the number of filled LHC bunches will be increased, allowing $\langle\mu>$ to be reduced, and hence maximizing the number of single $p p$ interactions in the $\mathrm{LHCb}$ region. The plan is then to run LHCb with almost constant luminosity, so-called "luminosity levelling", and up to a maximum intensity of $\sim 3 \times 10^{32} \mathrm{~cm}^{-2} \mathrm{~s}^{-1}$ (which is already $50 \%$ above design). We hope to collect $\geq 200 \mathrm{pb}^{-1}$ of data by the end of June and $\sim 1 \mathrm{fb}^{-1}$ by the end of 2011 .

\section{Summary}

LHCb running in 2010 has been highly successful, with $37 \mathrm{pb}^{-1}$ of data being recorded. The experiment has proven to be extremely versatile, operating under challenging conditions of multiple interactions per bunch crossing, whilst still retaining good detector efficiency. Despite only a small fraction of luminosity having been delivered, $\mathrm{LHCb}$ is already competing with the Tevatron in some areas of $B_{s}$ physics and in 2011 will surpass the $B$ factories. The 2010 data-set is a tantalizing taste of things to come. There will be a long and exciting programme over several years to explore the full potential of physics beyond the Standard Model.

\section{Acknowledgments}

I would like to thank our colleagues in the CERN accelerator departments for achieving the excellent performance of the LHC. I express my gratitude the members of the LHCb collaboration who have helped me prepare this presentation, with special mention to Andrei Goloutvin, Patrick Koppenburg, Marcel Merk, Olaf Steinkamp and Frederic Teubert. I would like to thank the conference organisers, in particular Robert Fleischer, Marcel Merk, Niels Tuning and Leo Wiggers, who made Beauty 2011 in Amsterdam so enjoyable. 
Finally, this paper is dedicated to the memory of Peter Schlein, Werner Ruckstuhl and Tom Ypsilantis. They contributed so much, yet sadly never got to see the results from this pioneering experiment.

\section{References}

[1] H1 Collaboration, F.D. Aaron et al., Measurement of Charm and Beauty Jets in Deep Inelastic Scattering at HERA. Eur. Phys. J. C71 1509 (2011).

ZEUS Collaboration, H. Abramowicz et al., Measurement of beauty production in deep inelastic scattering at HERA using decays into electrons. Eur. Phys. J. C71 1573 (2011).

[2] The HERA-B Collaboration, HERA-B: An experiment to study CP violation in the B system using an internal target at the HERA proton ring. Design Report, DESY-PRC-95-01, Jan 1995, 491pp.

[3] The LHCb Collaboration, A. Augusto Alves et al., The LHCb Detector at the LHC. JINST 3:S08005, (2008).

[4] The LHCb Collaboration, Roadmap for selected key measurements of LHCb. LHCB-PUB-2009-029, Dec 2009. 379pp. e-Print: arXiv:0912.4179.

[5] S. Vecchi. Flavour tagging and mixing at $L H C b$, these proceedings.

See also LHCb CONF-2011-03; LHCb-CONF-2011-10.

[6] V. Vagnoni, $B \rightarrow$ hh (hadronic final states), these proceedings.

[7] The LHCb Collaboration, R. Aaij et al., First observation of $B_{s} \rightarrow D_{s 2}^{+}(2573) \mu^{-} \bar{v}_{\mu}$ decays. Phys. Lett. B698 (2011) 14.

[8] The LHCb Collaboration, R. Aaij et al., First observation of $B_{s}^{0} \rightarrow J / \psi f_{0}(980)$ decays. Phys. Lett. B698 (2011) 115.

[9] The LHCb Collaboration, First observation of the decay $\bar{B}_{s} \rightarrow D^{0} K^{* 0}$. LHCb-CONF-2011-019

[10] The LHCb Collaboration, First observation of the decay $\bar{B}_{s} \rightarrow D^{0} K^{* 0}$. LHCb-CONF-2011-008.

[11] M. Gersabeck, Charm mixing and CP violation at $L H C b$, these proceedings. 